\title{
Level Correlations And Persistent Currents In Mesoscopic Metals
}

\author{
S. Sitotaw and R.A. Serota* \\ Department of Physics \\ University of Cincinnati \\ Cincinnati OH 45221-0011
}

\begin{abstract}
We use the exact correlation function of the density of energy levels in the magnetic field to evaluate persistent currents in mesoscopic metals. We also analyze the perturbation theory limit of the correlation function vis-a-vis the perturbation theory limit of the orbital response.
\end{abstract}

\section{INTRODUCTION}

Orbital magnetism and persistent currents in mesoscopic metal rings and grains have received much attention in the past decade. Theoretically, mesoscopic orbital magnetism is rather well understood for temperature $T \gg \Delta$, where $\Delta$ is the mean energy level spacing, and for magnetic fields such that the flux through the sample in $\phi \lesssim \phi_{0}=h c / e$ - the flux quantum [1] $\left(\phi_{0}=2 \pi / e\right.$ in units where $\left.\hbar=c=1\right)$. To evaluate the thermodynamics quantities at these temperatures, it is sufficient to characterize the energy spectrum in terms of the level density $\rho$. In particular, the orbital magnetic dependence of the level density correlation function,

$$
R\left(\omega, \tau_{H}{ }^{-1}\right)=\left\langle\delta \rho\left(\varepsilon_{1}, H\right) \delta \rho\left(\varepsilon_{2}, H\right)\right\rangle
$$

where $\delta \rho(\varepsilon, H)=\rho(\varepsilon, H)-\langle\rho\rangle$ and the brackets stand for disorder averaging, describes both the mean mesoscopic response and the fluctuations [1].

Furthermore, in this limit it is sufficient to use the perturbative limit of the correlation function. In the twoCooperon approximation, it is given by [1],

$$
R_{\text {pert }}\left(\omega, \tau_{H}^{-1}\right)=\frac{s^{2}}{2 \beta \pi^{2}} \operatorname{Re} \frac{1}{\left(-i \omega+\gamma+\tau_{H}^{-1}\right)^{2}}
$$

$\omega=\left(\varepsilon_{2}-\varepsilon_{1}\right), \gamma$ is the level broadening (which will be neglected below relative to $T$, as it should for metals), and $s$ and $\beta$ are integers whose values depend on the symmetry class. For Gaussian Orthogonal Ensemble (GOE) $\beta=1$, while for Gaussian Symplectic Ensemble (GSE) $\beta=4$ due to the fact that 3 out of 4 spin channels are eliminated by spin-orbit scattering. Neglecting Zeeman splitting, $s=2$ in either case due to double spin degeneracy and $\langle\rho\rangle \Delta=s$. $\tau_{H}^{-1}$ gives the orbital magnetic dependence. For a single period for a ring with the flux $\phi$, it is given by [1]

$$
\tau_{H}^{-1}=(2 \pi)^{2} E_{c}\left(\frac{2 \phi}{\phi_{0}}\right)^{2}
$$

where $E_{c}=\hbar \tau_{d i f f}^{-1} \gg T$ and $\tau_{d i f f}=L^{2} / D$ is the time of diffusion around the ring of circumference $L$ (time of diffusion to sample boundaries for simply connected geometries, such as disk (1]), $D$ being is the diffusion coefficient.

In the evaluation of the mean response one must remember that the electrons in a closed system form a canonical ensemble [2]. Ingeniously, Imry [3] found a simple relationship between the free energy of a canonical ensemble and the free energy and particle-number fluctuation of an equivalent grand canonical ensemble (see Appendix A). It was shown [4], however, that such a relationship holds only if the particle-number fluctuation is large, which is the case when $T \gg \Delta$ also. A perturbative evaluation [1] using Imry's formalism yields a persistent current $\propto T^{-1}$. The validity of this result is obviously limited to temperatures $T \gtrsim \Delta$.

Evaluation of the orbital magnetic response for $T<\Delta$ is a challenging problem. Unlike spin magnetism, which can be analyzed in terms of a few-level problem [5], all states of the Fermi sea contribute, in principle, and there are large cancellations between the diamagnetic and paramagnetic components. As a first step, however, one can use a "mixed approach" wherein Imry's formalism is combined with the exact form of the level-density correlation function. Such an approach should, hopefully, reveal a trend in the persistent current as $T$ becomes smaller than $\Delta$.

*serota@physics.uc.edu

${ }^{1}$ For a narrow ring, the response is $\phi_{0} / 2$ periodic so there is no need to consider larger fluxes. 
Two earlier attempts of this nature were undertaken in Refs. [6] and [7]. While both correctly argued that the apparent singularity for $T \rightarrow 0$ found in the perturbative approximation disappears for GOE as the persistent current saturates to a finite value as $T$ crosses $\Delta$, they bore serious deficiencies. The former did not find a closed form of the correlation function and considered only $T=0$ (while Imry's formalism, as was pointed out above, is rigorously applicable when $T \gg \Delta$ ). The latter considered the entire range of temperatures but the magnetic field dependence of the correlation function was crudely mimicked by a substitution $\gamma \rightarrow \gamma+\tau_{H}^{-1}$ (as in perturbative expression (2)) in the exact correlation function obtained in the assumption of presence of level broadening $\gamma$ alone. Later, however, the "mixed approach" was fully implemented for spin magnetism [4] where the magnetic field dependence of the level density correlation function is rather simple.

It turns out, however, that the exact correlation function in the presence of a time-reversal symmetry breaking field, such as magnetic field, had been calculated over a decade earlier by Mehta and Pandey [8] for both GOE and GSE using random matrix theory (RMT) (their result was subsequently confirmed using the supersymmetric non-linear $\sigma$-model in Ref. [9]). In this work we combine the exact correlation function obtained in Refs. [8] and [9] with Imry's formalism to find the magnetic field dependence of the persistent current for finite temperatures.

\section{LEVEL CORRELATION FUNCTION}

In units of $\langle\rho\rangle^{2}$, the dependence of the correlation function of the level density on the time reversal symmetry breaking parameter $\lambda$ in transition from GOE to GUE (Gaussian Unitary Ensemble) and GSE to GUE is given by 8:

$$
\begin{aligned}
R_{\text {orth }}(x, \lambda) & =\int_{1}^{\infty} d k \int_{0}^{1} d k^{\prime} \frac{k^{\prime}}{k} \sin (k x) \sin \left(k^{\prime} x\right) e^{-2 \lambda^{2} \pi^{2}\left(k^{2}-k^{\prime 2}\right)} \\
R_{\text {symp }}(x, \lambda) & =\frac{1}{4} \int_{1}^{\infty} d k \int_{0}^{1} d k^{\prime} \frac{k}{k^{\prime}} \sin (k x) \sin \left(k^{\prime} x\right) e^{-2 \lambda^{2} \pi^{2}\left(k^{2}-k^{\prime 2}\right)}
\end{aligned}
$$

respectively, where $x=\frac{\pi}{\Delta}\left|\varepsilon_{1}-\varepsilon_{2}\right|$. For a ring enclosing the flux $\phi$ [9],

$$
\lambda^{2}=\left(4 \pi \Delta \tau_{H}\right)^{-1}
$$

where $\tau_{H}{ }^{-1}$ is given by eq. (3).

It follows from eqs. (4) and (5) that

$$
\begin{aligned}
R_{\text {orth }}(x, 0) & =-\frac{d}{d x}\left(\frac{\sin (x)}{x}\right)\left(\frac{\pi}{2}-\mathrm{Si}(x)\right) \\
R_{\text {symp }}(x, 0) & =\frac{1}{4}\left(\pi \delta(x)+\frac{d}{d x}\left(\frac{\sin (x)}{x}\right) \operatorname{Si}(x)\right)
\end{aligned}
$$

The main difference between GOE and GSE is the $\delta$-function term in $R_{\text {symp }}(x, 0)$. More precisely, it is a $\lambda \rightarrow 0$ limit of 10

$$
\frac{\pi^{\frac{5}{2}} x^{2}}{4 \lambda^{\frac{3}{2}}} \exp \left(-\frac{\pi^{2} x^{2}}{4 \lambda}\right)
$$

The latter will prove to be responsible for the singular temperature dependence of the persistent current in GSE in the "mixed approach."

Consider now the full correlation function of the level density [8],

$$
\begin{aligned}
\mathcal{R}_{\text {orth }}(x, \lambda) & =\pi \delta(x)-\frac{\sin ^{2}(x)}{x^{2}}+R_{\text {orth }}(x, \lambda) \\
\mathcal{R}_{\text {symp }}(x, \lambda) & =\frac{1}{4}\left(\pi \delta(x)-\frac{\sin ^{2}(x)}{x^{2}}\right)+R_{\text {symp }}(x, \lambda)
\end{aligned}
$$

and notice that for either ensemble $R(x, \lambda) \rightarrow 0$ when $\lambda \rightarrow \infty$. It is clear then, using eqs. (7) and (8), that GOE, GSE, and GUE all exhibit a property of "level rigidity," as manifested by

$$
\int \mathcal{R}(x, 0) d x=\int \mathcal{R}(x, \infty) d x=0
$$

(in fact, it is likely that $\int \mathcal{R}(x, \lambda) d x=0$ for any $\lambda$ ). 


\section{PERSISTENT CURRENTS}

As was explained in Refs. [3] and [1], the dominant contribution to the mean orbital magnetic response of isolated metals is given by the part of the magnetic free energy which differentiates a canonical ensemble from an equivalent grand canonical ensemble (whose mean particle number coincides with the fixed particle number of a canonical ensemble). For $T \gg \Delta$, it is given by eq. (A9) of Appendix A,

$$
\begin{aligned}
\delta F_{H} & =\frac{1}{2\langle\rho\rangle} \partial_{H} \iint f\left(\varepsilon_{1}, \mu\right) f\left(\varepsilon_{2}, \mu\right) R\left(\varepsilon_{1}, \varepsilon_{2}, \tau_{H}{ }^{-1}\right) d \varepsilon_{1} d \varepsilon_{2} \\
& =-\frac{1}{2\langle\rho\rangle} \partial_{H} \int d \omega \frac{\omega}{\exp \left(\frac{\omega}{T}\right)-1} R\left(\omega, \tau_{H}{ }^{-1}\right)
\end{aligned}
$$

where integration on $\left(\varepsilon_{1}+\varepsilon_{2}\right)$ was performed. Once $\delta F_{H}$ is known, the persistent current and the magnetic moment are obtained, respectively via the following thermodynamic identities

$$
I=-\frac{\partial \delta F_{H}}{\partial \phi}, M=-\frac{\partial \delta F_{H}}{\partial H}
$$

We will use the expressions for the correlation function given in the previous Section to evaluate eqs. (13) and (14).

\section{A. Perturbative limit}

Here we address how the perturbation theory limit, whose leading term is given by eq. (2), is recovered from eqs. (4) and (5) (as was already mentioned, in what follows we will neglect $\gamma$ ). This limit is important for the evaluation of the orbital magnetic response when $T \gg \Delta$. The linear-response part, in particular, is defined by the term $\propto \tau_{H}^{-1}$ in the free energy. Notice, however, that the expansion of eq. (2) in orders of $\tau_{H}^{-1}$ yields no linear contribution. The contribution linear in $\tau_{H}^{-1}$ in the level correlation function comes, in fact, from the next order term in expansion of eqs. (4) and (5) and corresponds to the three-Cooperon diagrams, while it is the two-Cooperon diagrams that yield eq. (2). On the other hand, we will see that in the expression for the free energy such an expansion of the correlation function is not well defined and a careful analysis shows that the two-Cooperon diagrams give a leading contribution to the linear response, while the contribution of the three-Cooperon diagrams is smaller in the parameter $\Delta / T$.

Eq. (4) can be written as

$$
\begin{aligned}
R_{\text {orth }}(x, \lambda) & \approx \operatorname{Im}\left\{\int_{1} \frac{e^{\left(i x-4 \lambda^{2} \pi^{2}\right) k}}{k} d k\right\} \operatorname{Im}\left\{\int^{1} d k^{\prime} k^{\prime} e^{\left(i x+4 \lambda^{2} \pi^{2}\right) k^{\prime}}\right\} \\
& =\frac{1}{4}\left[e^{i x-4 \lambda^{2} \pi^{2}}\left(\frac{1}{i x-4 \lambda^{2} \pi^{2}}+\frac{1}{\left(i x-4 \lambda^{2} \pi^{2}\right)^{2}}\right)-c . c .\right]\left[e^{i x+4 \lambda^{2} \pi^{2}}\left(\frac{1}{i x+4 \lambda^{2} \pi^{2}}-\frac{1}{\left(i x+4 \lambda^{2} \pi^{2}\right)^{2}}\right)-\text { c.c. }\right]
\end{aligned}
$$

where we used the fact that for the large values of $\left|i x-4 \lambda^{2} \pi^{2}\right|$ the main contribution will come from $k, k^{\prime} \sim 1$, so that $k^{2} \approx 2 k-1, k^{\prime 2} \approx 2 k^{\prime}-1$ and used integration by parts. Omitting oscillating terms in eq. (15), we find

$$
R_{\text {orth }}(x, \lambda) \approx \frac{1}{2} \operatorname{Re}\left\{\frac{1}{\left(-i x+4 \lambda^{2} \pi^{2}\right)^{2}}-\frac{2}{\left(-i x+4 \lambda^{2} \pi^{2}\right)^{3}}\right\}
$$

Recalling that $\langle\rho\rangle \Delta=s$, we finally obtain

$$
R_{\text {orth }}\left(\omega, \tau_{H}{ }^{-1}\right) \approx \frac{s^{2}}{2 \beta \pi^{2}} \operatorname{Re}\left\{\frac{1}{\left(-i \omega+\tau_{H}^{-1}\right)^{2}}-\frac{2}{\left(-i \omega+\tau_{H}^{-1}\right)^{3}}\right\}, \beta=1
$$

A similar calculation yields

$$
R_{\text {symp }}\left(\omega, \tau_{H}^{-1}\right) \approx \frac{s^{2}}{2 \beta \pi^{2}} \operatorname{Re}\left\{\frac{1}{\left(-i \omega+\tau_{H}^{-1}\right)^{2}}+\frac{2}{\left(-i \omega+\tau_{H}{ }^{-1}\right)^{3}}\right\}, \beta=4
$$

The first term in eqs. (17) and 18 coincides with eq. (2) and the second term represents the three-Cooperon diagrams. 
Substituting eq. (2) in eq. (13), we find

$$
\delta F_{H}=-\frac{1}{2\langle\rho\rangle} \partial_{H} \int d \omega R_{\text {pert }}\left(\omega, \tau_{H}{ }^{-1}\right) \frac{\omega}{\exp \left(\frac{\omega}{T}\right)-1}
$$

Expansion of $R_{\text {pert }}$ in terms of $\tau_{H}{ }^{-1}$ would lead to divergence of the integral at zero. Consequently, one needs to treat carefully the second order pole at $-i \tau_{H}{ }^{-1}$. Alternatively, one can change the order of $\int$ and $R e$ and the pole will be of the first order. Using the latter procedure, one can close the contour in the plane that does not contain the pole and evaluate the integral in terms of the sum over Matsubara frequencies, $\omega_{m}=2 \pi i m T$. Since each summand is $\propto m$ and $\tau_{H}{ }^{-1}$ is finite at this stage of the evaluation, the $m=0$ term yields zero and the expansion in orders of $\tau_{H}{ }^{-1}$ is possible in the $m \geq 1$ Matsubara sum. As a result, one finds the formulae derived in Ref. [1]. The second term in eqs. (17) and (18) can be treated similarly and, as was mentioned above, gives a correction of the order of $\Delta / T$.

For completeness, a detailed perturbative evaluation of the persistent current is given in Appendix B. Its main conclusion is that the persistent current is given by

$$
\begin{aligned}
& I_{\text {pert }}(\phi) \approx \frac{s^{2}}{2 \beta \pi^{2}} \frac{8 \varsigma(2) e E_{c}}{T\langle\rho\rangle} \frac{\phi}{\phi_{0}}, \phi \ll \phi_{c} \\
& I_{\text {pert }}(\phi) \approx \frac{s^{2}}{2 \beta \pi^{2}} \frac{e}{2 \pi\langle\rho\rangle} \frac{\phi_{0}}{\phi}, \phi_{c} \ll \phi \ll \phi_{0}
\end{aligned}
$$

where

$$
\phi_{c} \sim \sqrt{\frac{T}{4 \pi E_{c}}} \frac{\phi_{0}}{2} \ll \phi_{0}
$$

is the magnetic flux scale for GOE to GUE transition. We emphasize the universal character of the decay of the persistent current with the flux in eq. (21) first noticed in Ref. [1].

\section{B. "Mixed approach"}

We now proceed to use the exact level correlation functions (4) and (5) in eq. (13). While the latter is valid for $T \gg \Delta$, in which case the perturbative expression (2) for the correlation function is sufficient, we hope that the results obtained in such an approach will be qualitatively indicative of the behavior for $T<\Delta$.

\section{Orthogonal ensemble}

For the orthogonal ensemble eq. (14) yields, with the help of eq. (6), the following expression for the persistent current

$$
\begin{aligned}
I_{\text {orth }}(\phi)= & \frac{\pi^{3}}{2} \frac{\partial \tau_{H}^{-1}}{\partial \phi} \frac{T^{2}\langle\rho\rangle}{\Delta} \\
& \sum_{m=1}^{\infty} m \int_{1}^{\infty} d k \int_{0}^{1} d k^{\prime} \frac{k^{\prime}}{k}\left(k^{2}-k^{\prime 2}\right)\left(e^{-2 \pi^{2} m \frac{T}{\Delta}\left(k-k^{\prime}\right)}-e^{-2 \pi^{2} m \frac{T}{\Delta}\left(k+k^{\prime}\right)}\right) e^{-\frac{1}{2} \pi\left(\Delta \tau_{H}\right)^{-1}\left(k^{2}-k^{\prime 2}\right)}
\end{aligned}
$$

where the integral over $\omega$ was reduced, by means of contour integration, to summation over the Matsubara frequencies $\omega_{m}=2 \pi m T, m=1,2,3 \ldots$. Performing summation over $m$, we obtain

$$
\begin{aligned}
I_{\text {orth }}(\phi)= & \frac{\pi^{3}}{8} \frac{\partial \tau_{H}^{-1}}{\partial \phi} \frac{T^{2}\langle\rho\rangle}{\Delta} \\
& \int_{1}^{\infty} d k \int_{0}^{1} d k^{\prime} \frac{k^{\prime}}{k}\left(k^{2}-k^{\prime 2}\right)\left(\frac{1}{\sinh ^{2}\left(\pi^{2} \frac{T}{\Delta}\left(k-k^{\prime}\right)\right)}-\frac{1}{\sinh ^{2}\left(\pi^{2} \frac{T}{\Delta}\left(k+k^{\prime}\right)\right)}\right) e^{-\frac{1}{2} \pi\left(\Delta \tau_{H}\right)^{-1}\left(k^{2}-k^{\prime 2}\right)}
\end{aligned}
$$

The integral in eq. (24) cannot be, in general, evaluated analytically and we proceed to analyze some limiting cases which can be explicitly handled. 
a. Perturbative limit, $T \gg \Delta$ While we have already discussed the perturbation theory limit, $T \gg \Delta$, it is tutorial to derive the latter directly from eq. (24). To this effect, omitting the exponentially small term with $\left(k+k^{\prime}\right)$ and taking into account the fact that the main contribution comes from $k, k^{\prime} \sim 1$, we find

$$
\begin{aligned}
I_{\text {orth }}(\phi) & \approx \frac{\pi^{3}}{4} \frac{T^{2}\langle\rho\rangle}{\Delta} \frac{\partial \tau_{H}^{-1}}{\partial \phi} \int_{1} d k \int^{1} d k^{\prime} \frac{\left(k-k^{\prime}\right)}{\sinh ^{2}\left(\pi^{2} \frac{T}{\Delta}\left(k-k^{\prime}\right)\right)} e^{-\pi\left(\Delta \tau_{H}\right)^{-1}\left(k-k^{\prime}\right)} \\
& =\frac{\pi^{3}}{4} \frac{T^{2}\langle\rho\rangle}{\Delta} \frac{\partial \tau_{H}^{-1}}{\partial \phi} \int_{0}^{\infty} d u \frac{u}{\sinh ^{2}\left(\pi^{2} \frac{T}{\Delta} u\right)} e^{-\pi\left(\Delta \tau_{H}\right)^{-1} u} \int_{-u}^{0} d v
\end{aligned}
$$

where we first shifted the variables of integration by 1 (since the integrand depends only on $k-k^{\prime}$ ) and then introduced the new variables $u=\left(k-k^{\prime}\right)$ and $v=k^{\prime}$ and extended the upper limit of integration to $\infty$ due to the exponentially decaying integrand. Re-scaling the variable of integration, $x=(\pi T / \Delta) u$, we finally arrive at eq. (B2) in Appendix B.

b. Non-perturbative limit, $T \ll \Delta$ Expanding the sinh-functions in eq. (24), we find

$$
I_{\text {orth }}(\phi) \approx \frac{s}{2 \pi} \frac{\partial \tau_{H}^{-1}}{\partial \phi} \int_{1}^{\infty} d k \int_{0}^{1} d k^{\prime} \frac{k^{\prime 2}}{\left(k^{2}-k^{\prime 2}\right)} e^{-\frac{1}{2} \pi\left(\Delta \tau_{H}\right)^{-1}\left(k^{2}-k^{\prime 2}\right)}
$$

This persistent current is the limiting current in the zero-temperature limit. Notice, that the upper limit of the integral on $k$ has been extended to $\infty$ (despite the small-parameter expansion of the integrand), which introduces a correction of order $\max \left\{\pi^{2} T / \Delta,\left(\frac{1}{2} \pi \tau_{H}^{-1} / \Delta\right)^{1 / 2}\right\}$ to eq. (26). As in the perturbative case, eq. (26) has the following limiting behaviors:

$$
\begin{aligned}
& I_{\text {orth }}(\phi) \approx \frac{s}{2 \pi} \frac{\partial \tau_{H}^{-1}}{\partial \phi}=8 e E_{c} \frac{\phi}{\phi_{0}}, \phi \ll \phi_{c} \\
& I_{\text {orth }}(\phi) \approx \frac{s^{2}}{2 \beta \pi^{2}} \frac{e}{2 \pi\langle\rho\rangle} \frac{\phi_{0}}{\phi}, \phi_{c} \ll \phi \ll \phi_{0}
\end{aligned}
$$

where now $\phi_{c}$ is found from the condition $\frac{1}{2} \pi \tau_{H}^{-1} / \Delta \sim 1$ as

$$
\phi_{c} \sim \sqrt{\frac{\Delta}{2 \pi^{3} E_{c}}} \frac{\phi_{0}}{2} \ll \phi_{0}
$$

and we used the fact that

$$
\int_{1}^{\infty} d k \int_{0}^{1} d k^{\prime} \frac{k^{\prime 2}}{\left(k^{2}-k^{\prime 2}\right)}=\frac{1}{2}
$$

and took explicitly $s=2$ in eq. (27). Notice that the universal decay of the persistent current with the flux for $\phi_{c} \ll \phi \ll \phi_{0}$ coincides with the perturbative result of eq. (21): this is obvious both on physical and mathematical grounds and comes from $k \sim k^{\prime} \sim 1$.

\section{Symplectic ensemble}

For GSE, we obtain, analogously to GOE, the following expression for the persistent current

$$
\begin{aligned}
I_{\text {symp }}(\phi)= & \frac{\pi^{3}}{8 \beta} \frac{\partial \tau_{H}^{-1}}{\partial \phi} \frac{T^{2}\langle\rho\rangle}{\Delta} \\
& \int_{1}^{\infty} d k \int_{0}^{1} d k^{\prime} \frac{k}{k^{\prime}}\left(k^{2}-k^{\prime 2}\right)\left(\frac{1}{\sinh ^{2}\left(\pi^{2} \frac{T}{\Delta}\left(k-k^{\prime}\right)\right)}-\frac{1}{\sinh ^{2}\left(\pi^{2} \frac{T}{\Delta}\left(k+k^{\prime}\right)\right)}\right) e^{-\frac{1}{2} \pi\left(\Delta \tau_{H}\right)^{-1}\left(k^{2}-k^{\prime 2}\right)}
\end{aligned}
$$

As for GOE, we proceed to investigate the limiting cases of this expression.

a. Perturbative limit, $T \gg \Delta$ This case is identical, up to coefficient $\beta^{-1}$, to the orthogonal ensemble since the reversed ratio $k / k^{\prime}$ relative to the latter is inconsequential in view of the fact that the main contribution to the integral comes from $k \sim k^{\prime} \sim 1$. As a result, we again find that the expression for the persistent current given in Appendix B, with the limiting behaviors described by eqs. (20) and (21). 
b. Non-perturbative limit, $T \ll \Delta$ As for GOE, expanding the sinh-functions in eq. (30), we find

$$
I_{\text {symp }}(\phi) \approx \frac{s}{2 \beta \pi} \frac{\partial \tau_{H}^{-1}}{\partial \phi} \int_{1}^{\infty} d k \int_{0}^{1} d k^{\prime} \frac{k^{2}}{\left(k^{2}-k^{\prime 2}\right)} e^{-\frac{1}{2} \pi\left(\Delta \tau_{H}\right)^{-1}\left(k^{2}-k^{\prime 2}\right)}
$$

which is the limiting current in the zero-temperature limit. We first address the zero-field limit. Towards this end, we notice that

$$
\int_{1}^{\infty} d k \int_{0}^{1} d k^{\prime} \frac{k^{2}}{\left(k^{2}-k^{\prime 2}\right)} e^{-\alpha\left(k^{2}-k^{\prime 2}\right)} \stackrel{\alpha \longrightarrow 0}{\longrightarrow} \frac{1}{2} \sqrt{\frac{\pi}{\alpha}}
$$

which can be easily obtained by differentiation on $\alpha$ and subsequent evaluation of the integral in terms of the Error Functions. Consequently, we obtain

$$
I_{\text {symp }}(0) \approx \frac{s}{2 \beta \pi} \frac{\partial \tau_{H}^{-1}}{\partial \phi} \frac{1}{2} \sqrt{\frac{2 \Delta}{\tau_{H}^{-1}}}=\frac{s e}{\beta \pi} \sqrt{2 \Delta E_{c}}, T=0
$$

The latter indicates the existence of the permanent current (and orbital magnetic moment) for GSE, as first conjectured by Kravtsov and Zirnbauer [10].

The permanent current in a zero flux implies a Curie-type response for non-zero temperature. To obtain the latter, we need to be more careful when expanding the sinh-functions in eq. (30). Namely, we start by expanding in $\pi^{2} \frac{T}{\Delta} k^{\prime} \ll 1$ which yields

$$
\begin{aligned}
I_{\text {symp }}(\phi) & \approx \frac{\pi^{5}}{2 \beta} \frac{\partial \tau_{H}^{-1}}{\partial \phi} \frac{T^{3}\langle\rho\rangle}{\Delta^{2}} \int_{1}^{\infty} d k \int_{0}^{1} d k^{\prime} k\left(k^{2}-k^{\prime 2}\right) \frac{\cosh \left(\pi^{2} \frac{T}{\Delta} k\right)}{\sinh ^{3}\left(\pi^{2} \frac{T}{\Delta} k\right)} e^{-\frac{1}{2} \pi\left(\Delta \tau_{H}\right)^{-1}\left(k^{2}-k^{\prime 2}\right)} \\
& \approx \frac{\pi^{5}}{2 \beta} \frac{\partial \tau_{H}^{-1}}{\partial \phi} \frac{T^{3}\langle\rho\rangle}{\Delta^{2}} \int_{1}^{\infty} d k k \frac{\cosh \left(\pi^{2} \frac{T}{\Delta} k\right)}{\sinh ^{3}\left(\pi^{2} \frac{T}{\Delta} k\right)} \int_{0}^{1} d k^{\prime}\left(k^{2}-k^{\prime 2}\right), \phi \ll \frac{\phi_{0}}{2} T \sqrt{\frac{2 \pi}{\Delta E_{c}}}
\end{aligned}
$$

where the latter condition on the flux gives the range of applicability of the linear response for GSE in the limit $T \ll \Delta$. Rescaling the variable of integration and omitting terms small in ratio $\Delta / T$, we find

$$
I_{\text {symp }}(\phi) \approx \frac{1}{2 \pi^{3} \beta} \frac{\partial \tau_{H}^{-1}}{\partial \phi} \frac{\Delta^{2}\langle\rho\rangle}{T} \int_{0}^{\infty} d y y^{3} \frac{\cosh (y)}{\sinh ^{3}(y)}=\frac{2 s e}{\beta} \frac{\Delta}{T} E_{c} \frac{\phi}{\phi_{0}}
$$

From the latter, $I_{\text {symp }}(\phi) \sim(s e / \beta) \sqrt{2 \pi \Delta E_{c}}$ at $\phi \sim\left(\phi_{0} / 2\right) T \sqrt{2 \pi /\left(\Delta E_{c}\right)}$ - in qualitative agreement with eq. (33).

\section{DISCUSSION}

The most interesting result is a possibility of a Curie-like orbital response in GSE at temperatures smaller than the mean energy-level spacing. In the "mixed approach" considered in this work, it appears as a result of broadening by the magnetic flux of a $\delta$-function term in the level density correlation function [10], in accordance with eq. (9). While this broadening was attributed [10] to lifting of the Kramers degeneracy [11] by the magnetic field, the level density description of the spectrum is, obviously, insensitive to the differences between even- and odd-electron systems (this equally applies to the use of eq. (13) which assumes large variations of the particle number in the equivalent grand canonical ensemble). On the other hand, the Kramers theorem distinguishes between even- and odd-electron systems. In the odd case, the Curie orbital response would be due to a permanent persistent current (moment) in a state "unmatched" by a time-reversed state of a degenerate doublet. The "mixed approach" would then mimic the average behavior between the even- and odd-electron systems. The situation is in complete analogy with spin magnetism where the "mixed approach" is indicative of the Curie response but one needs to study [4], [5] few-level systems at $T \ll \Delta$ to correctly account for odd/even differences. A similar analysis of the orbital response is complicated by the need to consider large Fermi sea cancellations between its diamagnetic and paramagnetic components. While odd/even difference is not important for GOE, since the Fermi level states are not current-carrying, the few-level analysis of the van-Vleck response must be carried out for $T \ll \Delta$. We will address these issues in a future work.

\section{ACKNOWLEDGMENT}

We thank Bernard Goodman for very useful discussions. 


\section{APPENDIX A: RESPONSE OF THE CANONICAL ENSEMBLE}

In this Appendix we derive the relationship between the free energies of the canonical ensmble and an equivalent grand canonical ensemble in the limit $T \gg \Delta$. Denoting $\partial_{H}$ the change of the appropriate quantities with the magnetic field, we find

$$
\partial_{H} F=\partial_{H} \Omega+N \partial_{H} \mu
$$

where it is assumed that the chemical potential of a system may depend, generally speaking, on the magnetic field. Using the idea that the magnetic field dependence can be transferred from the energy level dependence to the density of states [12],

$$
\Omega=-T \int d \varepsilon \rho(\varepsilon, H) \ln \left(1+\exp \left(\frac{\mu-\varepsilon}{T}\right)\right)
$$

we find

$$
\partial_{H} \Omega=-T \int d \varepsilon \partial_{H} \rho(\varepsilon, H) \ln \left(1+\exp \left(\frac{\mu-\varepsilon}{T}\right)\right)-\left(\partial_{H} \mu\right) N
$$

where

$$
N=\int d \varepsilon \rho(\varepsilon, H)\left(1+\exp \left(\frac{\varepsilon-\mu}{T}\right)\right)^{-1}
$$

is the fixed number of particles in the canonical ensemble and the mean number of particles in the equivalent grand canonical ensemble. Combining eqs. (A1) - (A4), we obtain

$$
\partial_{H} F=-T \int d \varepsilon \partial_{H} \rho(\varepsilon, H) \ln \left(1+\exp \left(\frac{\mu-\varepsilon}{T}\right)\right)
$$

where the $\left(\partial_{H} \mu\right) N$ term, containing the dependence of the chemical potential on the field, canceled out. Notice that this is in agreement with the small corrections theorem wherein the corrections to $F$ and $\Omega$ are the same, but in the latter we do not vary $\mu$. The derivation that follows is insensitive to the dependence of $\mu$ on the magnetic field but only to the fact that it varies from system to system [3].

Denoting $\langle\mu\rangle$ the average value of $\mu$ over all systems (disorder-average) in zero field, we proceed to expand in powers of $\mu-\langle\mu\rangle$

$$
\begin{aligned}
\partial_{H} F \approx & -T \int d \varepsilon \partial_{H} \rho(\varepsilon, H) \ln \left(1+\exp \left(\frac{\langle\mu\rangle-\varepsilon}{T}\right)\right) \\
& -(\mu-\langle\mu\rangle) \int d \varepsilon \partial_{H}(\rho(\varepsilon, H)-\langle\rho\rangle) f(\varepsilon,\langle\mu\rangle)
\end{aligned}
$$

where $f(\varepsilon,\langle\mu\rangle)$ is the Fermi distribution function and the first term has the meaning of $\partial_{H} \Omega(\langle\mu\rangle)$. Taking into account the following expression for the number of particles,

$$
\begin{aligned}
N & =\int d \varepsilon\langle\rho\rangle f(\varepsilon,\langle\mu\rangle) \\
& =\int d \varepsilon \rho(\varepsilon, H) f(\varepsilon, \mu) \\
& \approx \int d \varepsilon\langle\rho\rangle f(\varepsilon,\langle\mu\rangle)+\int d \varepsilon(\rho(\varepsilon, H)-\langle\rho\rangle) f(\varepsilon,\langle\mu\rangle)-(\mu-\langle\mu\rangle) \int d \varepsilon\langle\rho\rangle f^{\prime}(\varepsilon,\langle\mu\rangle)
\end{aligned}
$$

and the fact that the last integral is $-\langle\rho\rangle$, we obtain, comparing the first and the last lines of the above equation,

$$
(\mu-\langle\mu\rangle) \approx-\langle\rho\rangle^{-1} \int d \varepsilon(\rho(\varepsilon, H)-\langle\rho\rangle) f(\varepsilon,\langle\mu\rangle)
$$

Substituting the latter in eq. (A6) and performing disorder averaging, we find 


$$
\begin{aligned}
\delta F_{H} & \equiv \partial_{H} F-\partial_{H} \Omega(\langle\mu\rangle) \\
& \approx \frac{1}{\langle\rho\rangle} \iint d \varepsilon_{1} d \varepsilon_{2}\left\langle\delta \rho\left(\varepsilon_{1}, H\right) \partial_{H} \delta \rho\left(\varepsilon_{2}, H\right)\right\rangle f\left(\varepsilon_{1},\langle\mu\rangle\right) f\left(\varepsilon_{2},\langle\mu\rangle\right) \\
& =\frac{1}{2\langle\rho\rangle} \partial_{H} \iint d \varepsilon_{1} d \varepsilon_{2}\left\langle\delta \rho\left(\varepsilon_{1}, H\right) \delta \rho\left(\varepsilon_{2}, H\right)\right\rangle f\left(\varepsilon_{1},\langle\mu\rangle\right) f\left(\varepsilon_{2},\langle\mu\rangle\right) \\
& =\frac{1}{2\langle\rho\rangle} \partial_{H}\left\langle\delta N^{2}\right\rangle
\end{aligned}
$$

where $\left\langle\delta N^{2}\right\rangle$ is the mean particle number fluctuation in the equivalent grand canonical ensemble [13] described by $\Omega(\langle\mu\rangle)$.

The transformation in eq. (A9) can be explained as follows. Notice that $\left\langle\delta \rho\left(\varepsilon_{1}, H_{1}\right) \partial_{H_{2}} \delta \rho\left(\varepsilon_{2}, H_{2}\right)\right\rangle$ can be written as $\partial_{H_{2}}\left\langle\delta \rho\left(\varepsilon_{1}, H_{1}\right) \delta \rho\left(\varepsilon_{2}, H_{2}\right)\right\rangle$. However, the diffuson contribution to $\left\langle\delta \rho\left(\varepsilon_{1}, H_{1}\right) \delta \rho\left(\varepsilon_{2}, H_{2}\right)\right\rangle$ depends on $\left(H_{1}-H_{2}\right)^{2}$ and the Cooperon contribution on $\left(H_{1}+H_{2}\right)^{2}$ (see, for instance, Ref. [1]). Expanding in $\left(H_{1} \pm H_{2}\right)^{2}$ and taking the limit $H_{1} \rightarrow H_{2}=H$, we arrive at the desired result.

All of the above results can be also obtained in a purely thermodynamical framework using the small corrections theorem [13]. Indeed, the latter states that small corrections to all thermodynamic potentials are the same provided that the appropriate thermodynamic variables are kept constant. Therefore, we write,

$$
\delta_{H} F=\delta_{H} \Omega(\mu(H), H)
$$

an no variation of $\mu(H)$ is done in the r.h.s. This is in correspondence with eq. A9) where only the level density is varied with the field. Expanding around $\langle\mu\rangle$, we find

$$
\delta_{H} F \approx \delta_{H} \Omega(\langle\mu\rangle, H)+\delta_{H}\left(\frac{\partial \Omega}{\partial \mu}(\langle\mu\rangle, H)\right)(\mu-\langle\mu\rangle)
$$

which, again, is in complete correspondence with the expansion in eq. A6 . Noticing that

$$
N(\mu(H), H)=N
$$

we reproduce eq. (A9) (neglecting the difference between the thermodynamic and single-particle densities of states, $\partial N / \partial \mu$ and $\langle\rho\rangle$ respectively, and after some manipulations analogous to those in eq. (A7)) as follows:

$$
\begin{aligned}
\delta F_{H} & \equiv \delta_{H} F-\delta_{H} \Omega(\langle\mu\rangle, H) \\
& \approx-\delta_{H}(N(\langle\mu\rangle, H)-N(\mu(H), H))(\mu-\langle\mu\rangle) \\
& \approx \frac{\partial N(\langle\mu\rangle, H)}{\partial \mu}\left(\delta_{H}(\mu-\langle\mu\rangle)\right)(\mu-\langle\mu\rangle) \\
& \approx \frac{\langle\rho\rangle}{2} \delta_{H}(\mu-\langle\mu\rangle)^{2} \approx \frac{1}{2\langle\rho\rangle} \delta_{H}(N-\langle N\rangle)^{2}
\end{aligned}
$$

\section{APPENDIX B: PERSISTENT CURRENT IN THE PERTURBATIVE APPROXIMATION}

Using eqs. (2), 19) and (14) we find, upon reducing the integral to the Matsubara sum,

$$
I_{\text {pert }}(\phi) \approx-\frac{s^{2}}{2 \beta \pi^{2}} \frac{T^{2}}{2\langle\rho\rangle} \frac{\partial}{\partial \phi} \sum_{m=1}^{\infty} \frac{m}{\left[m T+\left(2 \pi \tau_{H}\right)^{-1}\right]^{2}}
$$

With the help of the identity $u^{-2}=\int_{0}^{\infty} t \exp (-u t) d t$ the latter can be re-written as

$$
\begin{aligned}
I_{\text {pert }}(\phi) & =-\frac{s^{2}}{2 \beta \pi^{2}} \frac{T^{2}}{2\langle\rho\rangle} \frac{\partial}{\partial \phi} \int_{0}^{\infty}\left(\sum_{m=1}^{\infty} m e^{-m T t}\right) t \exp \left(-\frac{\tau_{H}^{-1}}{2 \pi} t\right) d t \\
& =\frac{s^{2}}{2 \beta \pi^{2}} \frac{1}{2 T\langle\rho\rangle} \frac{\partial \tau_{H}^{-1}}{\partial \phi} \int_{0}^{\infty}\left(\frac{\pi x}{\sinh (\pi x)}\right)^{2} \exp \left(-\frac{\tau_{H}^{-1}}{T} x\right) d x
\end{aligned}
$$


which coincides with the respective equation [n Ref. [1]. From eq. (B2) it follows that [1]

$$
\begin{aligned}
& I_{\text {pert }}(\phi) \approx \frac{s^{2}}{2 \beta \pi^{2}} \frac{1}{2 \pi T\langle\rho\rangle} \frac{\partial \tau_{H}^{-1}}{\partial \phi} \int_{0}^{\infty}\left(\frac{x}{\sinh x}\right)^{2} d x=\frac{s^{2}}{2 \beta \pi^{2}} \frac{8 \varsigma(2) e E_{c}}{T\langle\rho\rangle} \frac{\phi}{\phi_{0}}, \phi \ll \phi_{c} \\
& I_{\text {pert }}(\phi) \approx \frac{s^{2}}{2 \beta \pi^{2}} \frac{1}{2 T\langle\rho\rangle} \frac{\partial \tau_{H}^{-1}}{\partial \phi} \int_{0}^{\infty} \exp \left(-\frac{\tau_{H}^{-1}}{T} x\right) d x=\frac{s^{2}}{2 \beta \pi^{2}} \frac{e}{2 \pi\langle\rho\rangle} \frac{\phi_{0}}{\phi}, \phi_{c} \ll \phi \ll \phi_{0}
\end{aligned}
$$

where $\varsigma(2)=\pi^{2} / 6$ and $\phi_{c}$ is found from the condition $\tau_{H}^{-1} \sim T$ and is given by [1]

$$
\phi_{c} \sim \sqrt{\frac{T}{4 \pi^{2} E_{c}}} \frac{\phi_{0}}{2} \ll \phi_{0}
$$

For completeness, we also derive the result accounting for the Aharonov-Bohm periodicity in narrow rings. This is easily accomplished by using the following expression for the perturbative level-density correlation function [14]

$$
R_{\text {pert }}\left(\omega, \tau_{H}{ }^{-1}\right)=\frac{s^{2}}{2 \beta \pi^{2}} \sum_{n=-\infty}^{\infty} \operatorname{Re} \frac{1}{\left(-i \omega+4 \pi^{2} E_{c}\left(n+\frac{2 \phi}{\phi_{0}}\right)^{2}\right)^{2}}
$$

As a result, we find the following expression for the persistent current

$$
I_{\text {pert }}(\phi) \approx-\frac{s^{2}}{2 \beta \pi^{2}} \frac{T^{2}}{2\langle\rho\rangle} \frac{\partial}{\partial \phi} \sum_{n=-\infty}^{\infty} \sum_{m=1}^{\infty} \frac{m}{\left[m T+2 \pi E\left(n+\frac{2 \phi}{\phi_{0}}\right)^{2}\right]^{2}}
$$

which can be evaluated using and identity

$$
\sum_{n=-\infty}^{\infty} \frac{1}{\left[b^{2}+(n+a)^{2}\right]}=\frac{\pi}{b} \frac{\sinh (2 \pi b)}{\cosh (2 \pi b)-\cos (2 \pi a)}
$$

(notice that for small $a$ and $b$ the sum is dominated by the $n=0$ term - in agreement with the procedure adopted in the bulk of the article). From eqs. ( $\mathrm{B} 7)$ and (B8), we find

$$
I_{\text {pert }}(\phi) \approx \frac{s^{2}}{2 \beta \pi^{2}} \frac{\pi T^{2}}{2\langle\rho\rangle E_{c}} \frac{\partial}{\partial T} \frac{\partial}{\partial \phi} \sum_{m=1}^{\infty} \frac{1}{\sqrt{\frac{2 \pi T}{E_{c}} m}} \frac{\sinh \left(\sqrt{\frac{2 \pi T}{E_{c}} m}\right)}{\cosh \left(\sqrt{\frac{2 \pi T}{E_{c}} m}\right)-\cos \left(\frac{4 \pi \phi}{\phi_{0}}\right)}
$$

In Ref. [1], the sum appearing in this equation was evaluated using the Euler-Maclaurin method. However, the latter does not work well for small values of the argument. Consequently, here we choose a different approach. Namely, we use the fact that $2 \pi T \ll E_{c}$ and expand the argument for $m \ll E_{c} / 2 \pi T$ and extend the sum to infinity thus neglecting the correction of the order $2 \pi T / E_{c}$

$$
\begin{aligned}
I_{p e r t}(\phi) & \approx \frac{s^{2}}{2 \beta \pi^{2}} \frac{2 e E_{c}}{\pi T\langle\rho\rangle} \sin \left(4 \pi \phi / \phi_{0}\right)\left[\psi^{(1)}(z)+\frac{1}{2} z \psi^{(2)}(z)\right] \\
z & =\frac{E_{c}}{\pi T}\left[1-\cos \left(4 \pi \phi / \phi_{0}\right)\right]
\end{aligned}
$$

where $\psi^{(n)}(z)$ is the polygamma function. Using $\psi^{(1)}(z)+\frac{1}{2} z \psi^{(2)}(z) \stackrel{z \longrightarrow 0}{\longrightarrow} \varsigma(2)$ and $\psi^{(1)}(z)+\frac{1}{2} z \psi^{(2)}(z) \stackrel{z \longrightarrow \infty}{\longrightarrow}(2 z)^{-1}$, we recover - in the limit $\phi \ll \phi_{0}$ - eqs. (B3) and (B4) from eq. (B10).

\footnotetext{
${ }^{2}$ Notice that, due to a misprint, the factor $T^{-1}$ is missing in eq. (18) of Ref. [1].
} 
[1] S. Oh, A.Yu. Zyuzin, and R.A. Serota, Phys. Rev. B44, 8858 (1991) and references therein.

[2] R. Kubo, J. Phys. Soc. Japan 17, 975 (1962).

[3] Y. Imry, in Coherence Effects in Condensed Matter Systems, edited by B. Kramer (Plenum, NY, 1991). See also A. Schmid, Phys. Rev. Lett. 66, 80 (1991); F. von Oppen and E. K. Riedel, ibid. 66, 84 (1991); B.L. Altshuler, Y. Gefen, and Y. Imry, ibid. 66, 88 (1991).

[4] S. Sitotaw and R.A. Serota, Physica Scripta 53, 23 (1996).

[5] S. Sitotaw and R.A. Serota, Physica Scripta 53, 521 (1996); Denton, B. Mühschlegel, and D.J. Scalapino, Phys. Rev. B7, $3589(1973)$

[6] A. Altland, S. Iida, A. Müller-Groeling, and H.A. Weidenmüller, Europhys. Lett. 20, 155 (1992).

[7] R.A. Serota and A.Yu. Zyuzin, Phys. Rev. B47, 6399 (1993) and ibid. B48, 17649 (1993).

[8] M.L. Mehta and A. Pandey, J. Phys. A16, 2655 (1983).

[9] A. Altland, S. Iida, and K.B. Efetov, J. Phys. A16, 3545 (1993).

[10] V.E. Kravtsov and M.R. Zirnbauer, Phys. Rev. B46, 4332 (1992).

[11] L.D. Landau and E.M. Lifshitz, Quantum Physics, (Pergamon Press, New York 1965)..

[12] R.B. Dingle, Proc. Roy. Soc. Lond. A211, 500 (1952) and ibid. 517 (1952).

[13] L.D. Landau and E.M. Lifshitz, Statistical Physics, (Pergamon Press, New York 1980)

[14] B.L. Altshuler and B.Z. Spivak, JETP 65, 343 (1987). 